\title{
Summary of: Content and quality of website information on the treatment of oral ulcers
}

FULL PAPER DETAILS

${ }^{1}$ UCLH NHS Foundation Trust, Eastman Dental Hospital, UCL Eastman Dental Institute, 256 Grays Inn Road, London, WC1X 8LD ${ }^{*}$ Correspondence to: Dr Richeal Ni Riordain Email: richeal.niriordain@gmail.com

Online article number E15

Refereed Paper - accepted 22 July 2014

DOI: $10.1038 /$ sj.bdj.2014.886

-British Dental Journal 2014; 217: E15

\author{
R. Ni Riordain ${ }^{* 1}$ and T. Hodgson ${ }^{1}$
}

\begin{abstract}
Aims and objective To determine the type, accuracy and content of information available on the Internet regarding the treatment of oral ulcers. Method The search engine Google was used to generate a list of top 100 websites about 'mouth ulcer treatment'. The websites were evaluated using the DISCERN instrument and the JAMA benchmarks. Whether the site displayed the Health on the Net (HON) seal was also recorded. Results The search yielded 1,460,000 sites on the Google website. Of the top 100 sites a total of 54 sites were suitable for analysis due to duplicate links and non-functioning links. Of the websites analysed, 31.5\% achieved all four JAMA benchmarks and 13\% achieved only one benchmark. No website receiving the maximum mark on the overall score and six websites received the lowest overall score regarding the DISCERN instrument. The questions with the poorest response score were 'Does it describe how the treatment choices affect overall quality of life?' and 'Does it provide support for shared decision-making?'. Only 7.4\% of the websites displayed the HON seal. Conclusion Diverse information regarding the treatment of oral ulcers is available on the Internet with variable accuracy levels based on both JAMA benchmarks and DISCERN.
\end{abstract}

\section{EDITOR'S SUMMARY}

'Eighty percent of Internet-using adults search the web for health-related info', say the authors of this paper. Eighty percent! This paper concerns the information available on the Internet specifically relating to oral ulcers and their treatment. I suspect that 'oral ulcers' is a term frequently entered into search engines as this condition can be a nasty, painful one; it would be interesting to determine how often people do seek information on this affliction. It must be common that patients would often attempt to seek quick treatment options without going to see a healthcare professional.

As often mentioned in this Journal, it is well-nigh impossible to police the web in any meaningful way. There is certainly also a question about whether we would even want to do so if we could. Thus, it is becoming increasingly important for us all to be aware of what is actually on the web and to learn to discern fact from fiction. We certainly know for a fact that patients will be looking for answers there.

Indeed, recently we published a paper investigating the sale of dental products, namely over-the-counter bruxism splints, on the Internet. ${ }^{1}$ In that case it was a question of products rather than treatment advice but at the end of the day it all comes down to the information provided to the patient enabling them to make a decision.

In this paper, the authors have done lots of the work for us in determining what websites are advising in relation to the treatment for oral ulcers, with 'marmite' and 'beer washes' amongst the more unusual discoveries. The paper also reminds us of the usefulness of the 'HON seal'. Health on the Net (HON; www.hon.ch) was founded in 1996 to: "encourage the dissemination of quality health information for patients and professionals and the general public, and to facilitate access to the latest and most relevant medical data through the use of the internet.' The HON seal certifies that the website upholds the HON code of conduct, and this could be a valuable and clear indicator for patients as to the trustworthiness of health information on the Internet.

However, the information on the Internet is far from static. It is hopeless to try to keep up so I feel that the most important advice to clinicians in this paper is around providing guidance and education to patients regarding the reliability of treatment advice on the Internet. Advising them how to tell the wheat from the chaff so to speak, appears to be the current role of the clinician in relation to the Internet.

The full paper can be accessed from the $B D J$ website (www.bdj.co.uk), under 'Research' in the table of contents for Volume 217 issue 7.

\section{Ruth Doherty Managing Editor DOI: 10.1038/sj.bdj.2014.887}

1. Wassell R W, Verhees L, Lawrence K, Davies S, Lobbezoo F. Over-the-counter (OTC) bruxism splints available on the Internet. Br Dent J 2014; 216: E24. 


\section{TO ACCESS THE BDJ WEBSITE TO READ THE FULL PAPER:}

- BDA Members should go to www.bda.org.

- Click the 'login' button on the right-hand side and enter your BDA login details.

- Once you have logged in click the 'BDJ' tab to transfer to the BDJ website with full access.

IF YOUR LOGIN DETAILS DO NOT WORK:

- Get a password reminder: go to www.bda.org, click the login button on the right-hand side and then click the forgotten password link.

- Use a recommended browser: we recommend Microsoft Internet Explorer or Mozilla Firefox.

- Ensure that the security settings on your browser are set to recommended levels.

IF YOU HAVE NOT YET SIGNED UP TO USE THE BDA WEBSITE:

- Go to www.bda.org/getstarted for information on how to start using the BDA website.

\section{COMMENTARY}

The majority of the population served by the readers of this Journal will probably seek information on dental and medical problems affecting them, their family, friends, and colleagues from the internet. However, as the present paper of $\mathrm{Ni}$ Riordain and Hodgson indicates there is a considerable lack of appropriate information concerning the treatment of mouth ulcers available on websites across the globe.

Oral ulceration is almost without exception painful but has causes that range from simple local trauma, to significant haematological, gastrointestinal or mucocutaneous disease(s) and finally malignancy. Some of the causes are not notably common and thus patients may be unable to readily obtain advice from their immediate clinical carers, thus they may seek advice from the Internet.

The present paper demonstrates that information provided by sites in the first few pages of a search of the World Wide Web may not be helpful as there is little provision of reliable (evidencebased) facts on the symptom/signs, how it might be managed and how patients can be supported in making appropriate decisions upon the treatment of oral ulceration. Of concern, but perhaps unsurprisingly, commercially allied websites were commonly encountered thus raising concerns of bias, while unfortunately few sites were affiliated to academic/medical or government services - who presumably are the ones who are most likely to generate the true facts on this disease and endeavour to ensure patients receive appropriate advice/treatment.

Regardless of our circumstances the internet has much to offer be it finding directions from $\mathrm{A}$ to $\mathrm{B}$, booking trips or arranging shopping. Each of these tasks can usually be undertaken reliably. However, it would seem that when individuals are in genuine need of advice as regards one aspect of oral health a search of the Internet may not be helpful. Indeed, as the present paper illustrates, there is a genuine risk that individuals may obtain the wrong information that could cause harm, delay diagnosis, negatively impact upon long term outcomes, increase concern and lessen quality of life.

Not all individuals use the Internet, but for those that do there is a clear need to improve the availability of evidencebased information that will allow a person to make the right choices about their disease and its impact upon them. However, the creation of appropriately resourced websites can be challenging (eg the need to regularly update information), costly and must be planned carefully (with notable involvement by public and patient groups). A possible starting point, at least with oral ulceration, could be to expand the present mouth ulcers section of NHS Choices (http://www.nhs.uk/conditions/mouthulcer/pages/introduction.aspx) to include information that ensures that this fully accords with the DISCERN instrument and then move forward with an expansion across all common causes of mouth ulcers. This might ensure that at least one publicly funded website is providing resources that allow individuals to make well informed decisions.

\section{Professor Stephen Porter} Institute Director and Professor of Oral Medicine UCL Eastman Dental Institute London

\section{IN BRIEF}

- Presents information regarding the content quality and type of web-based information for the treatment of oral ulceration.

- Explores the need for the clinician to provide direction to patients searching online for health information due to the questionable quality of some of the information available.

- Discusses shared decision-making in healthcare.

\section{AUTHOR QUESTIONS AND ANSWERS}

1. Why did you undertake this research?

The idea for this research stemmed from an interesting presentation at the European Academy of Oral Medicine Scientific Conference in Athens in 2012, regarding the management of recurrent aphthous stomatitis. Reference was made to the management options being adopted by patients following online searches. We though it important to explore the information available on the Internet, assessing the quality and determining websites that may provide appropriate health information to which patients could be directed.

\section{What would you like to do next in this} area to follow on from this work?

As an algorithm determines the websites generated in a Google search, we cannot hope to influence which websites feature prominently when an online search is carried out. We can therefore only hope to inform patients of the concerns regarding the quality and content of web-based health information. By providing detailed health information to patients during consultations and links to further online information we can empower patients to become more involved in their own healthcare. We hope to use the findings from this study to provide such information for our patients with oral ulceration. 